\title{
Assisted Tunneling of a metastable state between barriers
}

\author{
G. Kälbermann* \\ Soil and Water dept., Faculty of Agriculture, Rehovot 76100, Israel
}

April 25, 2022

\begin{abstract}
The assisted tunneling of a metastable state between barriers is investigated analytically by means of a simplified one dimensional model. A time dependent perturbation changes the pole spectrum of the wave function introducing a larger decay constant. New insights about the decay of a metastable state are found. The scheme is exemplified for parameters corresponding to the nuclear process of $\alpha$ decay.
\end{abstract}

PACS 74.30.Gk, 73.43.Jn 23.60.+e

*e-mail address: hope@vms.huji.ac.il,germankal@hotmail.com 


\section{Introduction}

Quantum tunneling is the conventional theoretical paradigm for the explanation of a gamut of atomic, molecular and nuclear phenomena. Classically forbidden regions can be accessed by a quantum object whose behavior is described in terms of a wave function.

Shortly after the advent of quantum mechanics, Gurney and Condon and simultaneously Gamow, explained the huge differences between $\alpha$ decay lifetimes of similar unstable nuclei using the concept of tunneling through a barrier.[1,2] This framework has remained as reliable today as when it was proposed almost a century ago. [3, 4] The question of assisting tunneling, i.e. accelerating or decelerating the decay process is of the utmost importance in the nuclear context and elsewhere. In the nuclear case, acceleration of the decay process could change dramatically the treatment of radioactive waste as well as providing alternative fuel sources.

Analytical expressions for the various tunneling processes are extremely important. It is virtually impossible to follow numerically the evolution of a wave function to the long times involved in decay processes. There is a huge gap between the natural time scale of nuclear phenomena of the order of $10^{-24} \mathrm{sec}$ and the decay time scale of the order of milliseconds to millions of year.[6] The situation is somewhat, but not radically different, for atomic phenomena.

Not surprisingly, assisted tunneling is extensively investigated for solid state systems. The main theoretical tool in this endeavor is the Floquet formalism[7]. This method involves large matrices and it is computationally quite intensive. [8]. Other methods such as the "elevator effect" model of resonance assisted activation attempt to attack the problem in a semi-analytic manner[10]. Ivlev[9] has recently developed a complex time method to investigate assisted tunneling, aimed at a nonperturbative approximate treatment of the tunneling through special barriers.

There seems to be a clear consensus, that exposing a system to external excitations, time harmonic or not, can enhance its tunneling rate. The absence of exact analytical results, and reliable long term numerical results, especially for the nuclear case, makes difficult the assessment of experimental feasibility of implementing assisted activation.

In the present work we investigate an extremely simple model of a one dimensional quantum mechanical system located initially between fixed barriers. A time harmonic potential such as a low frequency -as compared to the natural frequency of the system- electric field, is then applied. The simplicity of the model allows a full, albeit perturbative, exact analytical solution. The perturbation causes a qualitative change in the pole structure of the wave function. The spectrum of poles arising from the normalization of the wave function that rule the decaying behavior of the metastable state, is extended. These poles in the complex momentum space, appear as the wave function is expanded in a complete set of the unperturbed system. The poles contribute in a contour integration of the wave amplitudes in momentum space.[4]. They are not introduced ad-hoc, as Gamow did with his complex energy method, but, arise in a systematic expansion of the wave function naturally. The time development of the wave function is dominated by the lowest energy poles. The intro- 
duction of the perturbation opens a new line of poles. Here again only one of them appears important. This new pole produces a bigger decay constant.

The result is not specific to time varying fields. The new line of poles arises even for a static spatially inhomogeneous potential such as that of a constant electric field. In a nuclear setting, such a static field will be certainly almost completely screened by the atomic electrons, whereas for a time varying potential, the efficiency of such screening is diminished. A discussion of the electronic screening problem is beyond the scope of the present work. We henceforth treat only a time harmonic perturbation that vanishes in the zero frequency limit.

The external vibration shakes the system back and forth between barriers and accelerates the tunneling process. We analyze the general and, mostly qualitative results, based on the pole spectrum. New insights into some troubling aspects of metastable state decay are offered in section 5. Extensive mathematical details will be presented in a forthcoming work.

\section{The Model}

The Schrödinger equation for the one dimensional system located in between barriers is taken to be

$$
i \frac{\partial \Psi}{\partial t}=\frac{-1}{2 m} \frac{\partial^{2} \Psi}{\partial x^{2}}+\lambda\left(\delta\left(x+x_{0}\right)+\delta\left(x-x_{0}\right)\right) \Psi
$$

Comparing to a finite size square barrier, $\lambda$ represents the height multiplied by the width. For nuclear $\alpha$ decay $\lambda \approx 2, m \approx 20 \mathrm{fm}^{-1}, x_{0} \approx 10 \mathrm{fm}, m \lambda x_{0}=400>>1$

We choose as initial nonstationary wave, a gaussian wave packet. This allows a full analytical treatment. In $\alpha$ decay, it would correspond to a preexisting spatially symmetric state.

$$
\Psi(x, t=0)=N e^{-\frac{x^{2}}{\Delta^{2}}}
$$

with $\mathrm{N}$ a constant factor normalization, and $\Delta$ is the width parameter of the packet. Inside the barrier region the packet disperses if $\Delta<x_{0}$. , but, soon enough it stops dispersing due to the presence of the barriers. It can then spread only through the tunneling process governed by the barriers.

The even (e) and odd (o) stationary states of eq.(1) are readily found to be

$$
\begin{gathered}
n_{e}(k) \chi_{e}(x)=\left\{\begin{aligned}
\cos (k x) & \text { if }|x|<x_{0} \\
A \cos (k x) \pm B \sin (k x) & \text { if } x>x_{0} \text { or } x<-x_{0}
\end{aligned}\right. \\
n_{o}(k) \chi_{o}(x)=\left\{\begin{aligned}
\sin (k x) & \text { if }|x|<x_{0} \\
\pm C \cos (k x)+D \sin (k x) & \text { if } x>x_{0} \text { or } x<-x_{0}
\end{aligned}\right.
\end{gathered}
$$


The set of even-odd functions is orthonormal and complete. The normalization factors ${ }^{1}$ are extremely important and determine the location of the poles in the complex plane. As shown below, these poles govern the exponential decay of metastable wave functions.

The normalization factors are

$$
\begin{aligned}
\left(n_{e}(k)\right)^{2} & =\pi\left(A(k)^{2}+B(k)^{2}\right) \\
A(k) & =1-\sin \left(2 k x_{0}\right) \frac{m \lambda}{2 k} \\
B(k) & =\frac{m \lambda}{k}\left(\cos \left(k x_{0}\right)\right)^{2} \\
k^{2}\left(n_{e}(k)\right)^{2} & =\pi\left(\left(k-\sin \left(2 k x_{0}\right) \frac{m \lambda}{2}\right)^{2}+\left(m \lambda\left(\cos \left(k x_{0}\right)\right)^{2}\right)^{2}\right) \\
\left(n_{o}(k)\right)^{2} & =\pi\left(C(k)^{2}+D(k)^{2}\right) \\
C(k) & =-\frac{m \lambda}{k}\left(\sin \left(k x_{0}\right)\right)^{2} \\
D(k) & =1+\sin \left(2 k x_{0}\right) \frac{m \lambda}{2 k} \\
k^{2}\left(n_{o}(k)\right)^{2} & =\pi\left(\left(k+\sin \left(2 k x_{0}\right) \frac{m \lambda}{2}\right)^{2}+\left(m \lambda\left(\sin \left(k x_{0}\right)\right)^{2}\right)^{2}\right)
\end{aligned}
$$

The time harmonic potential perturbation reads

$$
V(x, t)=\mu x \sin (\omega t)
$$

with $\mu$ a coupling constant. For an external electric field of intensity $E_{0}$ interacting with an $\alpha$ particle of charge $2\left|q_{e}\right|, \mu=2\left|q_{e}\right| E_{0}$.

The wave function is expanded in the complete set of even and odd states

$$
\Psi(x, t)=\sum_{i=e, o} \int_{0}^{\infty} \chi_{i}(k, x) a_{i}(k, t) e^{\frac{-i k^{2} t}{2 m}} d k
$$

dots denoting derivatives with respect to $t$.

The Schrödinger equation for the amplitudes $a_{e, o}$ becomes

$$
\begin{aligned}
& i \dot{a}_{e}(k)=\int e^{-\frac{\left(k^{\prime 2}-k^{2}\right) t}{2 m}}<\chi_{e}(k, x)|V(x, t)| \chi_{o}\left(k^{\prime}, x\right)>d k^{\prime} a_{o}\left(k^{\prime}, t\right) \\
& i \dot{a}_{o}(k)=\int e^{-\frac{\left(k^{\prime 2}-k^{2}\right) t}{2 m}}<\chi_{o}(k, x)|V(x, t)| \chi_{e}\left(k^{\prime}, x\right)>d k^{\prime} a_{e}\left(k^{\prime}, t\right)
\end{aligned}
$$

\footnotetext{
${ }^{1}$ The determination of the normalization and the completeness issue will be dealt with in an extended version of the paper.[5]
} 
The leading contribution to the matrix element of the interaction can be evaluated exactly

$$
\begin{aligned}
<\chi_{e}(k, x)|V(x, t)| \chi_{o}\left(k^{\prime}, x\right)> & =\int_{-\infty}^{\infty} \chi_{e}(k, x) V(x, t) \chi_{o}\left(k^{\prime}, x\right) d x \\
& \approx \mu \pi \frac{1}{n_{e}(k) n_{o}\left(k^{\prime}\right)} \frac{\partial \delta\left(k-k^{\prime}\right)}{\partial k^{\prime}} \sin (\omega t)
\end{aligned}
$$

Inserting eq.(10) in eq.(9) we obtain

$$
\begin{aligned}
& i \dot{a}_{e}(k, t)=-\frac{\pi}{n_{o}(k) n_{e}(k)}\left(a_{o}^{\prime}(k, t)-n_{o}^{\prime}(k) / n_{o}(k) a_{o}(k, t)-\frac{i k t}{m} a_{o}(k, t)\right) \mu \sin (\omega t) \\
& i \dot{a}_{o}(k, t)=-\frac{\pi}{n_{o}(k) n_{e}(k)}\left(a_{e}^{\prime}(k, t)-n_{e}^{\prime}(k) / n_{e}(k) a_{e}(k, t)-\frac{i k t}{m} a_{e}(k, t)\right) \mu \sin (\omega t)
\end{aligned}
$$

primes denoting derivatives with respect to $k$.

Rescaling to dimensionless variables

$$
\begin{aligned}
k & \rightarrow \tilde{k}=\frac{k}{\sqrt{m \omega}} \\
t & \rightarrow \tilde{t}=\omega t \\
\mu & \rightarrow \tilde{\mu}=\frac{\mu}{\sqrt{\omega^{3} m}}
\end{aligned}
$$

eq.(11) becomes

$$
\begin{aligned}
& i \dot{a}_{e}(\tilde{k}, \tilde{t})=-\tilde{\mu} \frac{\pi}{n_{o}(k) n_{e}(\tilde{k})}\left(a_{o}^{\prime}(\tilde{k}, \tilde{t})-n_{o}^{\prime}(\tilde{k}) / n_{o}(\tilde{k}) a_{o}(\tilde{k}, \tilde{t})-i \tilde{k} \tilde{t} a_{o}(\tilde{k}, \tilde{t})\right) \sin (\tilde{t}) \\
& i \dot{a}_{o}(\tilde{k}, \tilde{t})=-\tilde{\mu} \frac{\pi}{n_{o}(\tilde{k}) n_{e}(\tilde{k})}\left(a_{e}^{\prime}(\tilde{k}, \tilde{t})-n_{e}^{\prime}(\tilde{k}) / n_{e}(\tilde{k}) a_{e}(\tilde{k}, \tilde{t})-i \tilde{k} \tilde{t} a_{e}(\tilde{k}, \tilde{t})\right) \sin (\tilde{t})
\end{aligned}
$$

primes denoting derivatives with respect to $\tilde{k}$ and dots representing derivatives with respect to $\tilde{t}$.

$\tilde{\mu}$ is the relevant parameter of the problem. It is small for external angular frequencies $\omega_{\min }^{3}>\mu^{2} m .{ }^{2}$ For lower frequencies, nonperturbative solutions are required. The smaller the electric field, the lower the frequency for which the perturbative solution will be appropriate.

The amplitudes of eq.(13) are further expanded in powers of $\tilde{\mu}$

\footnotetext{
${ }^{2}$ For example, in $\alpha$ decay case with $\mu \approx 1 \mathrm{eV}^{2}$ corresponding to a strong electric field amplitude of $E_{0} \approx 510^{6} \mathrm{Volt} / \mathrm{m}, \omega_{\min }>10^{12} \mathrm{sec}^{-1}$.
} 


$$
\begin{aligned}
& a_{e}(\tilde{k}, \tilde{t})=\sum_{n=0}^{\infty} b_{e}^{(n)}(\tilde{k}, \tilde{t}) \tilde{\mu}^{n} \\
& a_{o}(\tilde{k}, \tilde{t})=\sum_{n=1}^{\infty} b_{o}^{(n)}(\tilde{k}, \tilde{t}) \tilde{\mu}^{n}
\end{aligned}
$$

with initial conditions

$$
\begin{aligned}
b_{e, o}^{(n)}(\tilde{k}, t=0) & =0 \text { for } n \geq 1 \\
b_{e}^{(0)}(\tilde{k}, \tilde{t}) & =b_{0}(\tilde{k}) / n_{e}(\tilde{k})=\int_{-\infty}^{\infty} N e^{-\frac{x^{2}}{\Delta^{2}}} \chi_{e}(k) d x
\end{aligned}
$$

We extracted the normalization factor of the even wavefunctions $n_{e}(k)$ for the sake of convenience. It is straightforward to show that all the odd $n$ powers of $a_{e}(\tilde{k}, \tilde{t})$ vanish identically, and the same is true for the even $n$ powers $a_{o}(\tilde{k}, \tilde{t})$. The substitution of eqs.(14) into eq.(13) produces a separate equation for each order $n$. The equations are integrable exactly order by order, although the expressions of higher order amplitudes become increasingly involved.

\section{Poles of the Wave Function}

Figure 1 shows $\frac{\pi}{k^{2} n_{e}(k)^{2}}$, and $\frac{\pi}{k^{2} n_{p}(k)^{2}}$, for the parameters $m \lambda x_{0}=400, x_{0}=10 \mathrm{fm}$. The thin and tall spikes are due to the extreme closeness of the minima of the normalization factors to their complex zeroes.

The amplitudes of eq.(14) determine the wave function through eq.(8). Integration over $k$ is dominated by the pole structure of the normalization factors $n_{o, e}(k)$.

The normalization factors of eq. $(5,6)$ are even in the argument $k$. Consequently, the behavior around their zeroes can be expressed in the form

$$
k^{2} n_{e, o}^{2} \approx \gamma_{e, o}^{(j)}\left(k^{2}-k_{e, o}^{(j)^{2}}\right)^{2}+\beta_{e, o}^{(j)}
$$

where $j$ enumerates the pole number.

For the $\delta$ barriers we have chosen, and in the limit of $m \lambda x_{0}>>1$, we can find simple expressions for the locations of the poles as well as the value of the norm factors on the real axis at $k=k_{e, o}^{(j)}$.

$$
\begin{aligned}
k_{e}=\frac{(2 n+1) \pi m \lambda}{2\left(1+m \lambda x_{0}\right)} & k_{o}=\frac{n \pi m \lambda}{\left(1+m \lambda x_{0}\right)} \\
\gamma_{e}=\pi \frac{2 m \lambda x_{0}^{3}\left(m \lambda x_{0}+4\right)}{(2 n+1)^{2} \pi^{2}} & \gamma_{o}=\pi \frac{m \lambda x_{0}^{3}\left(m \lambda x_{0}+4\right)}{4 n^{2} \pi^{2}} \\
\beta_{e}=\frac{(2 n+1)^{4} \pi^{4}}{16 m^{2} \lambda^{2} x_{0}^{4}} & \beta_{o}=\frac{n^{4} \pi^{4}}{m^{2} \lambda^{2} x_{0}^{4}}
\end{aligned}
$$



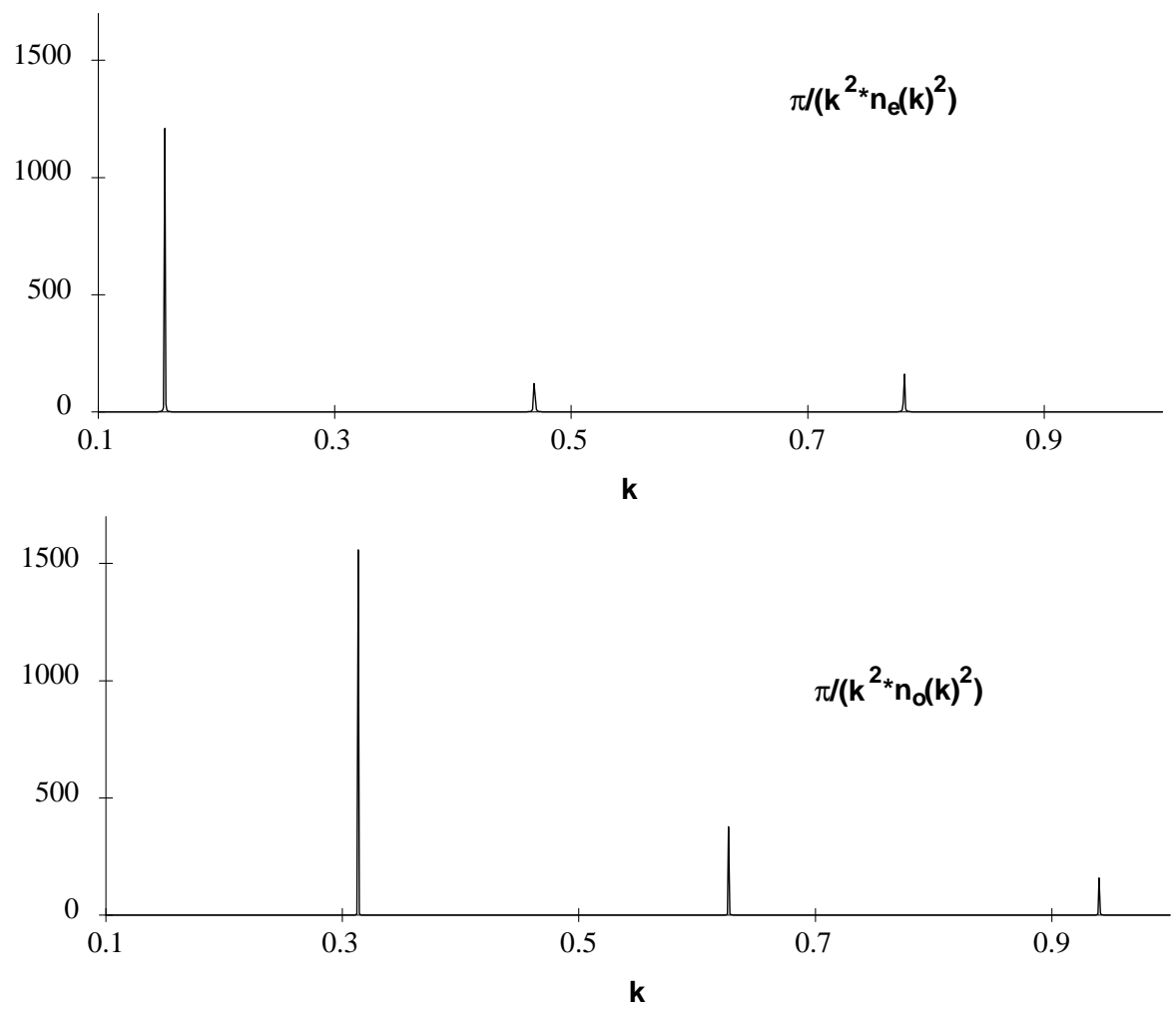

Figure 1: $\frac{\pi}{k^{2} n_{e}(k)^{2}}$, and $\frac{\pi}{k^{2} n_{p}(k)^{2}}$, as a function of $k$ in units of $\mathrm{fm}^{-1}$ for the parameters $m \lambda x_{0}=400, x_{0}=10 \mathrm{fm}$. 
The poles $q_{n}, q_{m}$ are located symmetrically above and below the real momentum axis at $k x_{0}$ approximately equal to an odd multiple of $\frac{\pi}{2}$ for the even case and, and a multiple of $\pi$ for the odd case, where the bound states of the infinite wall case lay.

$$
\begin{aligned}
& q_{n}^{e}=\left(\frac{(2 n+1) \pi m \lambda}{2\left(1+m \lambda x_{0}\right)}\right)^{2} \pm i \sqrt{\frac{\beta_{e}}{\gamma_{e}}} \\
& q_{o}^{2}=\left(\frac{n \pi m \lambda}{\left(1+m \lambda x_{0}\right)}\right)^{2} \pm i \sqrt{\frac{\beta_{o}}{\gamma_{o}}}
\end{aligned}
$$

The imaginary parts of the poles are orders of magnitude smaller than the real parts. For example, inserting the parameters corresponding to $\alpha$ decay, the first even pole appears at $q_{e, n=1} \approx 0.024 \pm i 3 * 10^{-7} \mathrm{fm}^{-2}$. For a finite size width, the imaginary parts are much smaller. The sharp spikes seen in figure 1 lead to the dominance of the poles in the spectrum of momenta.

When eq.(8) is evaluated by contour integration in the complex $k^{2}$ plane, the contour has to be closed from below. In the lower half-plane the convergence is insured by the exponential $e^{-i \frac{k^{2} t}{2 m}}$.

The poles are separated from each other, their contribution adds up. The negative imaginary part of each pole induces a time decaying exponential. Each exponent determines a different decay constant and decay time. If the original wave function is even in space, as in the expression of eq.(2), only even poles contribute to the unperturbed decay process. Due to the initial confinement of the wave to the inter-barrier region, the most important contribution arises from the first even pole. The influence of higher order poles is hindered by the wave packet transform to momentum space

$$
\Psi(k) \sim e^{-\frac{k^{2} \Delta^{2}}{4}}
$$

Therefore, the first pole drives the decay process and determines the lifetime of the quasistationary state.

\section{Acceleration of the Decay Process}

The structure of the solution of eq.(11) for the amplitude series of eq.(14) and to the lowest order in $\mu$ for the even amplitude is found to be

$$
\begin{aligned}
a_{e}(\tilde{k}, \tilde{t}) & =n_{e}(\tilde{k}) b_{0}(\tilde{k})\left(\frac{1}{n_{e}^{2}}+\tilde{\mu}^{2} \tilde{b}_{e}^{(2)}(\tilde{k}, \tilde{t})\right) \\
\tilde{b}_{e}^{(2)}(\tilde{k}, \tilde{t}) & =\frac{e_{1}(\tilde{t})+e_{2}(\tilde{t}) \tilde{k}^{2}}{n_{e}^{4} n_{o}^{2}}+\frac{e_{3}(\tilde{t}) \tilde{k} n_{o}^{\prime}}{n_{e}^{4} n_{o}^{3}}
\end{aligned}
$$




$$
\begin{aligned}
& +\frac{\tilde{k} e_{4}(\tilde{t}) n_{e}^{\prime}+e_{5}(\tilde{t}) n_{e}^{\prime \prime}}{n_{e}^{5} n_{o}^{2}} \\
& +\frac{e_{6}(\tilde{t})}{n_{e}^{6} n_{o}^{2}}+\frac{e_{7}(\tilde{t}) n_{e}^{\prime} n_{o}^{\prime}}{n_{e}^{5} n_{o}^{3}}
\end{aligned}
$$

The odd amplitude reads

$$
\begin{aligned}
a_{o}(\tilde{k}, \tilde{t}) & =n_{o}(\tilde{k}) b_{0}(\tilde{k}) \tilde{\mu} \tilde{b}_{o}^{(1)}(\tilde{k}, t) \\
\tilde{b}_{o}^{(1)}(\tilde{k}, \tilde{t}) & =\frac{k o_{1}(\tilde{t})}{n_{e}^{2} n_{o}^{2}}+\frac{o_{2}(\tilde{t}) n_{e}^{\prime}}{n_{e}^{3} n_{o}^{2}}
\end{aligned}
$$

where we have extracted a normalization factor in advance of the integration over the evenodd set of wave functions $\chi(k)$, as we want to identify the influence of the pole structure on the amplitudes. In eqs. $(20,21)$ we have suppressed the $\tilde{k}$ dependence of the normalization factors in the denominators for brevity.

We can readily analyze the structure of the perturbed amplitudes. The amplitudes in eqs. $(21,20)$ differ from the unperturbed amplitude $b_{0}(k)$ by the appearance of poles for the odd set of wave functions introduced by powers of $n_{o}(k)$ in the denominators.

The first even pole of eq.(17) generates a decay constant of the form

$$
\begin{aligned}
\left|e^{-i \frac{k_{e, n=1}^{2} t}{2 m}}\right| & \rightarrow e^{-\Lambda_{e} t} \\
\Lambda_{e} & =\frac{1}{2 m} \sqrt{\frac{\beta_{1}}{\gamma_{1}}} \\
& =\frac{\pi^{3}}{8 m x_{0}^{4} m^{2} \lambda^{2}}
\end{aligned}
$$

whereas the first odd pole generates a decay constant

$$
\Lambda_{o}=8 \Lambda_{e}
$$

The factor of 8 is specific to the $\delta$ model. In general there should be roughly this order of magnitude increase in the decay constant.

At the same time, the real part of the pole that enters the wave function mainly through the momentum space transform of the initial wave function of eq.(19) changes also

$$
\begin{aligned}
& e^{-\frac{\pi^{2} \Delta^{2}}{16 x_{0}^{2}}} \rightarrow e^{-\frac{\pi^{2} \Delta^{2}}{4 x_{0}^{2}}} \\
& e^{-\frac{\pi^{2} \Delta^{2}}{16 x_{0}^{2}}} \rightarrow e^{-\frac{9 \pi^{2} \Delta^{2}}{16 x_{0}^{2}}}
\end{aligned}
$$


The left hand side in both lines in eq.(24) correspond to the first even pole contribution whereas the right hand sides pertain to the first odd pole and the second even pole respectively.

There arise then two effects: An enhancement of the decay constant by a factor of 8 and, a damping factor. A rough estimate of the importance of the news terms can now be performed.

If initially $\Delta \approx x_{0}$, then the first odd pole contributes to the wave function about $10 \%$, while the second even pole contributes much less than 1\%. Even without getting into the cumbersome details of the wave function, the survival probability in the inner region between barriers will be influenced markedly by the introduction of the perturbation. The wave will tunnel faster.

\section{Flux Continuity}

The question of conservation of probability or flux continuity seems unavoidable at this junction. This problem arises even before adding a harmonic perturbation. If the wave diminishes with time everywhere it appears as if unitarity is broken. This should not occur in the evolution of a wave function with the Schrödinger equation and a real potential. The contradiction arises in other conventional approaches to the decay of a metastable state[4]. In the scattering method the paradox is apparently resolved by exhibiting another piece in the wave function that increases exponentially with time.

However, in the present treatment of the problem, the wave function receives contributions from the poles in the lower momentum half-plane only. There seems to be no time increasing piece at all. The exponentials originating from those poles is decreasing in time. On the other hand, flux has to be continuous and overall probability should be conserved. The answer to this conundrum brings to the fore the mathematical beauty of the decay process.

Consider the two separate regions, the inside zone around $x \approx 0$ and the long distance zone of $x \rightarrow \infty$, far away from the microscopic location of the barriers. In the inner region the integration over momentum space is dominated essentially by the poles. This is true until very long times. At very long times the exponential factor $e^{-i \frac{k^{2} t}{2 m}}$ oscillates wildly for small changes in the momentum. The integral of eq.(8) will start to suppress the importance of the pole share in the integral. The pole spike will be twisted by the oscillating factor with alternating positive and negative parts. It is possible to estimate the time at which the assumption of pole dominance fails as follows. The width of the pole spikes read off from eq.(16) is $\delta\left(k^{2}\right) \approx \gamma$. For $k^{2} \approx \gamma$ the inverse of the normalization factor essentially vanishes $\frac{1}{n_{o, e}^{2}} \rightarrow 0$. The pole dominance will end when

$$
\frac{k^{2} t}{2 m} \rightarrow \frac{\gamma t}{2 m} \approx 1
$$


Inspection of the values of $\gamma$ listed in eq.(17), it is found that this time is of the same order

of the lifetime $\frac{1}{\Lambda}$. Hence, in the inner region, the wave will decay until a time of the order of the lifetime. After that time, other contributions to the wave arising from momenta outside the poles have to be included. The wave leaks out of the inner region for very long times of the order of the lifetime of the state.

The behavior in the long distance region is completely different, even for short times. For $x \rightarrow \infty$ the oscillations in the integrand of eq.(8) stem from the harmonic functions in space $\chi$ of eqs. $(3,4)$. For the long distance region, the poles will never dominate, neither at short times nor at asymptotically long times. The wave is built from the whole momentum spectrum. Moreover, as time passes and, in the spirit of the stationary phase approximation, smaller and smaller momenta will dominate. This effect will enhance the wave at long distances, due to the momentum space factors of eq.(19). Consequently, the wave function will decay in the inner region and grow in the outer region and probability will be conserved. The same is true when more than one decay constant is present.

\section{Conclusions}

The investigation of assisted tunneling carried out in the present work implies that, there are distinctive qualitative features induced by the introduction of a time dependent perturbation. The main signal for the process is the appearance of another decay constant, bigger than the one of the unperturbed case. The state decays as if it were composed of two channels with different partial decay widths. A large component with a longer lifetime and a small, but presumably non-negligible component, with a much smaller -eight times smaller for $m \lambda x_{0}>>1$ - lifetime. Evidently, the fine details are important for an accurate determination of the wave function, and will be shown in a coming publication. The reverse process of tunneling into a region enclosed between barriers could be affected by the mechanism of assisted tunneling also. Such a possibility is of relevance for atomic and nuclear processes, such as nuclear fusion.

\section{References}

[1] R. W. Gurney and E.U. Condon, Phys. Rev. 33,127 (1929).

[2] G. Gamow, Zeit. Phys. 51, 204 (1928).

[3] E. L. Medeiros et al. Jour. of Phys. G32, B23 (2006).

[4] B. Holstein, Am. J. Phys. 64, 1062 (1996).

[5] M. Trott., S. Trott and Ch. Schnittler, Phys. Stat. Sol. B151 K123 (1989).

[6] W. Van Dijk and Y. Nogami, Phys. Rev. Lett. 83, 2867 (1999) 
[7] J. H. Shirley, Phys. Rev138, B979 (1965).

[8] M. Grifoni and P. Hänggi, Phys. Repts. 304, 229 (1998).

[9] J.P.Palomares-Báez, B. Ivlev and J. L. Rodriguez-López, Phys. Rev. A76, 05213 (2007), and references therein.

[10] M. Ya. Azbel, Phys. Rev. Lett. 68, 98 (1992). 